\title{
Gross and fine motor function in fibromyalgia and chronic fatigue syndrome
}

\author{
Omid Rasouli ${ }^{1,2}$ \\ Egil A Fors ${ }^{3}$ \\ Petter Chr Borchgrevink ${ }^{4,5}$ \\ Fredrik Öhberg ${ }^{6}$ \\ Ann-Katrin Stensdotter ${ }^{1}$ \\ 'Department of Neuromedicine \\ and Movement Science, Faculty \\ of Medicine and Health Sciences, \\ Norwegian University of Science \\ and Technology, Trondheim, Norway; \\ ${ }^{2}$ Department of Public Health \\ and Nursing, Faculty of Medicine \\ and Health Sciences, Norwegian \\ University of Science and Technology, \\ Trondheim, Norway; ${ }^{3}$ Department of \\ Public Health and Nursing, General \\ Practice Research Unit, Norwegian \\ University of Science and Technology, \\ Trondheim, Norway; ${ }^{4}$ Department of \\ Circulation and Medical Imaging, Pain \\ and Palliation Research Group, Faculty \\ of Medicine and Health Sciences, \\ Norwegian University of Science \\ and Technology, Trondheim, Norway; \\ ${ }^{5}$ National Competence Centre for \\ Complex Symptom Disorders, St. \\ Olav's University Hospital, Trondheim, \\ Norway; ${ }^{6}$ Department of Radiation \\ Sciences, Biomedical Engineering, \\ Umeå University, Umeå, Sweden
}

Correspondence: Omid Rasouli Faculty of Medicine and Health Sciences, Norwegian University of Science and Technology, Tungasletta 2, 749I,

Trondheim, Norway

Tel +47 73559277

Fax +4773559150

Email omid.rasouli@ntnu.no
This article was published in the following Dove Press journal:

Journal of Pain Research

7 February 2017

Number of times this article has been viewed

Purpose: This paper aimed to investigate motor proficiency in fine and gross motor function, with a focus on reaction time (RT) and movement skill, in patients with fibromyalgia (FM) and chronic fatigue syndrome (CFS) compared to healthy controls (HC).

Methods: A total of 60 individuals (20 CFS, 20 FM, and $20 \mathrm{HC}$ ), age 19-49 years, participated in this study. Gross motor function in the lower extremity was assessed using a RT task during gait initiation in response to an auditory trigger. Fine motor function in the upper extremity was measured during a precision task (the Purdue Pegboard test) where the number of pins inserted within $30 \mathrm{~s}$ was counted.

Results: No significant differences were found between FM and CFS in any parameters. FM and CFS groups had significantly longer RT than HC in the gait initiation ( $p=0.001$, and $p=0.004$ respectively). In the Purdue Pegboard test, $20 \%$ in the FM group, 15\% in the CFS groups, and $0 \%$ of $\mathrm{HC}$ group, scored below the threshold of the accepted performance. However, there were no significant differences between FM, CFS, and HC in this task $(p=0.12)$.

Conclusion: Compared to controls, both CFS and FM groups displayed significantly longer RT in the gait initiation task. Generally, FM patients showed the worst results in both tests, although no group differences were found in fine motor control, according to the Purdue Pegboard test. Keywords: fatigue syndrome, chronic, musculoskeletal diseases, gait initiation, Purdue Pegboard, reaction time

\section{Introduction}

Clinical studies have reported that fibromyalgia (FM) and chronic fatigue syndrome (CFS) are poorly distinguished from each other. ${ }^{1}$ Patients are usually diagnosed according to established clinical criteria ${ }^{2,3}$ because specific biological markers are lacking. ${ }^{4} \mathrm{FM}$ is characterized foremost by chronic widespread pain and CFS by chronic debilitating fatigue. However, it has been reported that $50 \%-70 \%$ of the signs and symptoms are common between these two groups of patients. ${ }^{5,6}$ Other common features are cognitive complaints $^{7}$ which may trouble these patients more than other symptoms. ${ }^{8}$ Memory and other cognitive abilities are dependent on attention and mental swiftness. Also, special consideration has been directed toward attention and processing speed of information. Impaired working memory and attention have been shown in patients with FM, ${ }^{9}$ while conflicting results about working memory ${ }^{10}$ and attention deficits ${ }^{11,12}$ have been published on CFS patients, and these may be as a result of using different protocols.

The processing speed of information is crucial not only for cognitive processes ${ }^{13,14}$ but also essential for motor control and fast and accurate motor responses such as 
pressing a computer key in response to visual stimuli. ${ }^{11,15}$ Such a performance is intrinsically dependent on perception and processing of somatosensory information. Longer response time in simple reaction time (RT) tasks has been demonstrated in both patient groups ${ }^{11,15}$ as has reduced psychomotor function, resulting in longer RT, as well as lower movement speed in tasks with higher cognitive demands. ${ }^{16,17}$

Tests of fine motor control for manual dexterity have uncovered deficits in patients with $\mathrm{FM},{ }^{18,19}$ but no tests of fine motor control appear to have been performed in patients diagnosed with CFS. Tests on gross motor control have revealed reduced gait velocity and bradykinesia ${ }^{20}$ and walking with altered muscle activation patterns in patients with FM. ${ }^{21}$ Similarly, lower self-selected gait velocity is reported in CFS. ${ }^{22}$ Moreover, previous studies suggest slowness in motor function in FM as well as CFS, but different studies have used different protocols, and fine motor skill appears to have not been investigated in CFS. Importantly, reduced motor speed in upper and lower extremity does seem to be interdependent, at least in the healthy elderly. ${ }^{23}$ As there is a discussion as to whether similarities in FM and CFS are signs of comorbidity or whether pain and fatigue are variations of symptoms in a common underlying disorder, patients with FM and CFS need to be subjected to the same test protocol in order to answer that question. ${ }^{1}$

The aim of the present study was to examine similarities and dissimilarities between FM and CFS for motor proficiency in gross and fine motor function, focusing on RT and speedaccuracy trade-off. A healthy group was included for comparison. We hypothesized that both patient groups would display similar deficits in psychomotor speed and control for fine as well as gross motor function compared to the control group. Motor functions in the lower and upper extremities were assessed by gait initiation and the Purdue Pegboard test, respectively.

\section{Material and methods}

\section{Study design}

This was a cross-sectional, observational, case-control study.

\section{Participants}

Forty patients and 20 healthy individuals participated in this study. All patients were diagnosed at the Department of Pain and Complex Disorders at the University Hospital. Twenty patients were diagnised with CFS (with no comorbidity of FM) according to US Centers for Disease Control and Prevention criteria, ${ }^{2}$ and 20 patients were diagnised with FM (with no comorbidity of CFS) according to the American College of Rheumatology (ACR) 1990 criteria. ${ }^{24}$ Patients were informed about the study at the clinic. Those interested were referred by their attending physician to participate and received an appointment for testing in our movement laboratory. In addition, 20 healthy controls (HC) matched for age and gender were recruited from staff and students at the university and hospital by announcement on the intranet. Only females were recruited as the majority of those diagnosed with CSF or FM are women. Pain and fatigue was rated in all participants with a numerical rating scale on the day of testing upon arrival to the laboratory, as the daily condition is known to vary in people in general, and these patient groups in particular, and may thus influence performance. To assess symptom severity, CFS patients also completed the Chalder Fatigue Scale $^{25}$ and the Fibromyalgia Impact Questionnaire (FIQ) ${ }^{26}$ in the lab before the testing was commenced. Characteristics of participants are described in Table 1. Exclusion criteria for all participants included diagnoses of major psychiatric disorders, neurological or musculoskeletal pathology (except chronic widespread pain for the CFS and FM group), injury, or uncorrected reduced vision. Verbal and written information was given, and written informed consent was obtained from each participant. The study was approved by the Regional Committees for Medical and Health Research Ethics (2012/679/REK midt) and conducted in accordance with the Declaration of Helsinki.

\section{Data acquisition}

\section{Gross motor function}

Proficiency in gross motor function was tested for the lower extremity with a RT task during gait initiation, ${ }^{27}$ based on the findings of bradykinesia in FM and CFS. The participant stood still and relaxed on a force platform, without shoes, and feet in parallel with self-selected distance between them. To create a baseline for the center of pressure $(\mathrm{CoP})$ measurement, 3 to

Table I Characteristics of the participants by group

\begin{tabular}{lllll}
\hline Variables & HC & CFS & FM & p-values \\
\hline Age (years) & $34.7(7.4)$ & $31.8(8.2)$ & $36.7(8.4)$ & 0.195 \\
Weight $(\mathrm{kg})$ & $69(9.5)^{\mathrm{a}}$ & $71.5(13.9)$ & $81.4(16.7)^{\mathrm{a}}$ & 0.014 \\
Height $(\mathrm{cm})$ & $167.2(7.5)$ & $168.2(5.6)$ & $169.7(6)$ & 0.455 \\
BMI $\left(\mathrm{kg} / \mathrm{m}^{2}\right)$ & $24.7(3)$ & $25.4(5.6)$ & $28.2(5.3)$ & 0.054 \\
Education (years) & $16.2(2.2)^{\mathrm{a}, \mathrm{b}}$ & $13.7(2.4)^{\mathrm{b}}$ & $13.8(2.0)^{\mathrm{a}}$ & $0.00 \mathrm{I}$ \\
Pain & $0.1(0.3)^{\mathrm{a}}$ & $0.9(1.2)^{\mathrm{b}}$ & $3.3(1.6)^{\mathrm{a}, \mathrm{b}}$ & $0.00 \mathrm{I}$ \\
Fatigue & $0.7(0.8)^{\mathrm{a}, \mathrm{b}}$ & $2.7(1.5)^{\mathrm{b}}$ & $2.6(1.9)^{\mathrm{a}}$ & $0.00 \mathrm{I}$ \\
Handedness (Rt/Lt) & $20 / 0$ & $20 / 0$ & $18 / 2$ & \\
Start leg (Rt/Lt) & $14 / 6$ & $16 / 4$ & $17 / 3$ & \\
\hline Notes: & & &
\end{tabular}

Notes: Data shown as mean (standard deviation) or number. ${ }^{a, b}$ Significant differences between groups are indicated by the same superscript letters.

Abbreviations: HC, healthy control; CFS, chronic fatigue syndrome; FM, fibromyalgia; BMI, body mass index; Rt, right; Lt, left. 
$5 \mathrm{~s}$ of steady state was recorded in each trial for quiet standing before an auditory trigger signal was issued. The delay time before release of the trigger was random and intentionally unpredictable. The participant was asked to initiate gait as fast as possible in response to the auditory signal (a beep), and then walk normally across a $3 \mathrm{~m}$ long walkway level with the force platform. The starting foot (left or right) was self-selected and kept the same throughout all trials. The test was repeated five times with a 1-minute rest between each trial. Participants had one trial practice to get accustomed to the test. RT was calculated using the CoP data collected with a Kistler force platform (type 9260AA6; Kistler Instrumente AG, Winterthur, Switzerland). The auditory signal was generated with a trigger button connected to an A/D board. All data were sampled and stored with the QTM software (Qualisys Track Manager, Gothenburg, Sweden; 2.10, build 2084) for further analysis.

\section{Fine motor function}

Following the gait initiation task, speed-accuracy of fine motor skill was assessed for the upper extremity with the Purdue Pegboard test (Model \#32020; Lafayette Instruments, Lafayette, IN, USA). The test was implemented according to the instruction in the Lafayette manual, starting with a short $10 \mathrm{~s}$ trial to ensure the task was understood. Only the first test in the test battery was performed. It consisted of placing pins into a row of 25 holes moving from top to bottom on the board placed on a table in front of the participant. The dominant hand was determined according to the question in the Edinburgh Handedness Inventory, and the test was performed once. For the right-handed individuals, the right side, and for the left-handed, the left side of the Purdue Pegboard was used. The number of inserted pins (size $\sim 2.54$ by $0.30 \mathrm{~cm}$ ) into the Pegboard in $30 \mathrm{~s}$ was counted. Average performance with the preferred hand in individuals $<60$ years of age is between $16-19$ pins. A score below 14 pins is considered a poor result. Reliability and validity have been established for the Purdue Pegboard across various population groups ${ }^{28}$ and it is considered as a relevant test for psychomotor skills and hand dexterity. ${ }^{29}$ The choice of the test was motivated by the background of a cognitive slowness in patients with FM and CFS.

\section{Data analyses}

Data were exported to MATLAB (R2015b) for analysis. An automated algorithm was used to detect the auditory signal and determine the first mediolateral deviation of CoP from baseline in quiet standing (Figure 1). ${ }^{30}$ The automated onsets were inspected and adjusted manually when necessary.
Validity of manual adjustments was validated by intertester reliability, performed by two independent and blinded investigators, to decide the onset of CoP in a random sample of 50 trials across groups. Cronbach's alpha was 0.985 for an average score and 0.970 for a single score. An Excel-matrix with onset times for deviation of $\mathrm{CoP}$ relative to the auditory signal (onset-time of the CoP deviation minus onset-time for trigger) was generated in MATLAB. For the Purdue Pegboard test, raw scores of the inserted pins were stated.

\section{Statistical analyses}

Statistical analyses were performed in SPSS (Version 22; IBM Corporation, Armonk, NY, USA). Data were inspected for normal distribution by graphical inspection of residuals in P-P and Q-Q plots and for skewness and kurtosis with histograms. Normal distribution was assessed statistically by Shapiro-Wilk test. Descriptive results of the RT and Purdue Pegboard test are presented as means \pm standard deviation (SD). There were no significant differences between the groups regarding age, height, and body mass index (BMI) (Table 1). Weight did however differ significantly between $\mathrm{HC}$ and FM, and there was a significant correlation between body weight of the participants and RT ( $r=0.42, p=0.001)$. Thus, weight was added as the covariate into the analysis. For RT, the mean value of five trials was calculated for each participant, and this was used for further analysis. The effect of group on RT was calculated using analysis of covariance (ANCOVA) with group ( $\mathrm{n}=3$; HC, CFS, and FM) as the between-subjects factor and weight as covariate. There was no correlation between weight and raw scores in the Pegboard test ( $r=-0.17, p=0.197$ ). Thus, a separate one-way ANOVA was conducted to compare raw scores in the Purdue Pegboard test between the groups ( $\mathrm{n}=3$; HC, CFS, and FM). We also ran separate one-way ANOVA on pain and fatigue scores. Pairwise comparisons with Bonferroni correction were performed to identify significant differences between the groups. Moreover, Fisher's exact test was performed to identify the differences in the proportions ( $<14$ pins) of inserted pins between the three groups. The correlation between different variables was investigated using Pearson correlation. The alpha level of significance was set at $P<0.05$.

\section{Results}

Table 1 displays the characteristics of participants. The mean score of FIQ was 54.7 (13.27) in the FM group, and the analysis showed no correlation between FIQ score and RT $(r=-0.24)$ or inserted pins $(r=0.30)$ in these patients $(p>0.05)$. The mean score of Chalder Fatigue Scale was 25.2 (3.57) in 


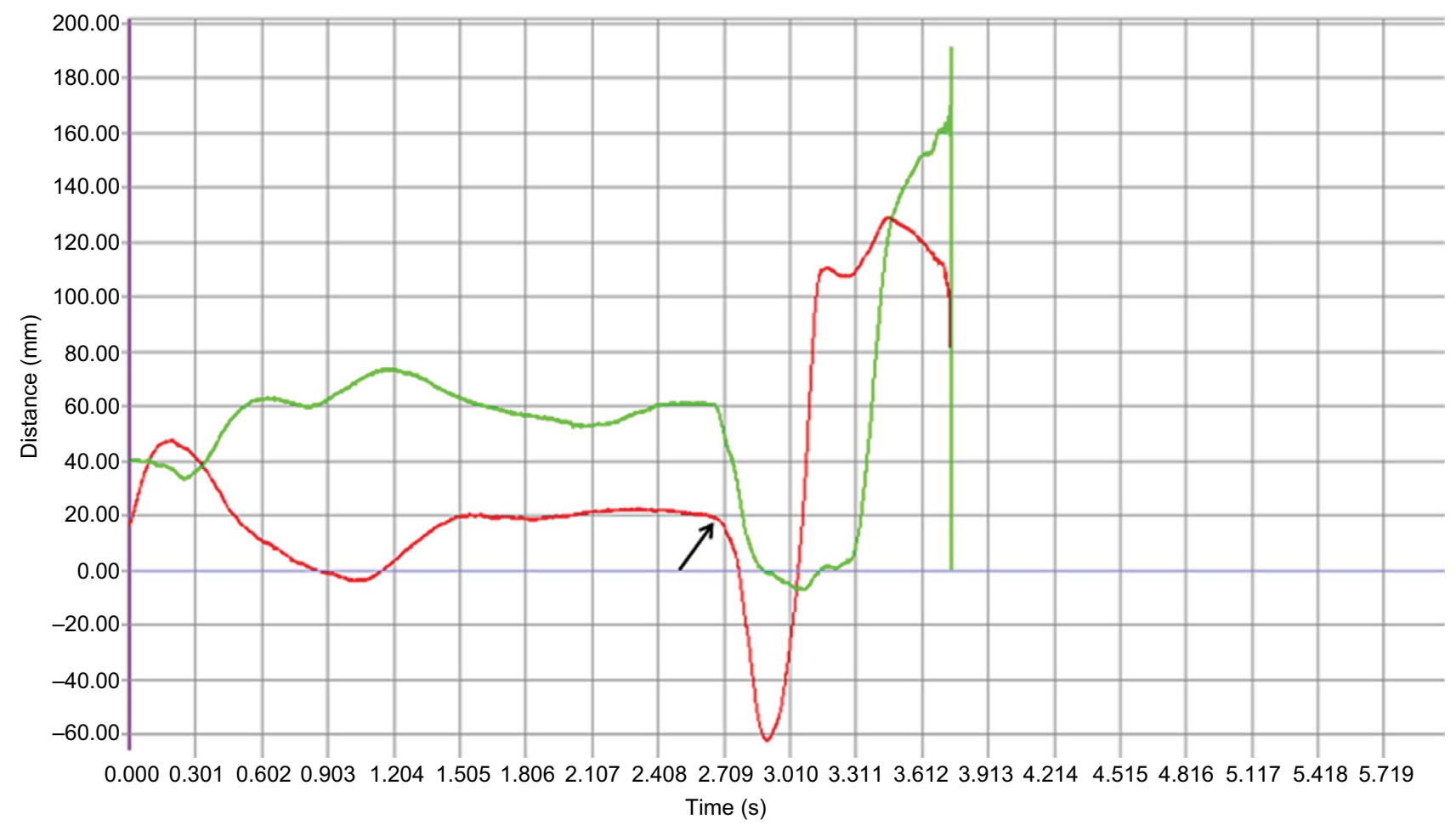

Figure I The onset determination of gait initiation.

Notes: The onset of gait initiation was determined from CoP as the first mediolateral deviation from baseline (black arrow), which occurred toward the same side as the swing leg. The red line shows mediolateral direction of CoP. The green line shows anteroposterior direction of CoP. At lift off of the swing leg, CoP deviated toward the side of the stance leg. A negative value denotes right direction. Thus, the right leg is the swing leg in this example. This pattern was uniform and occurred in all participants regardless of whether they were starting with the right or left leg.

Abbreviation: CoP, center of pressure.

the CFS patients, and, similarly, no correlation was found between Chalder score and RT ( $r=-0.18)$ or inserted pins $(r=0.05)$ in this group $(p>0.05)$.

\section{Gait initiation}

A total of 300 trials were recorded and used for the analysis. The FM group had the longest mean RT followed by CFS and $\mathrm{HC}$, which were 0.164 (0.02), 0.192 (0.02), and 0.205 (0.03) $\mathrm{s}$, respectively. A significant main effect of group, $\mathrm{F}(2,56)$ $=9.28, p<0.001$ was found indicating different RT at least in one group. The effect size was $\eta^{2}=0.25$, and observed power $=0.97$ for the group factor. Bonferroni-adjusted pairwise comparisons between groups showed that both the CFS and FM had significantly longer RT than HC (Table 2).

Table 2 Between-group comparisons of the RT results with Bonferroni adjustment

\begin{tabular}{lllll}
\hline Groups & Mean difference & Lower Cl & Upper Cl & p-values \\
\hline CFS - HC & 0.026 & 0.007 & 0.046 & $0.004^{*}$ \\
FM-HC & 0.034 & 0.013 & 0.054 & $0.00 I^{*}$ \\
FM-CFS & 0.007 & -0.013 & 0.027 & 1.000 \\
\hline
\end{tabular}

Notes: The participants' weight was used as the covariate. *Indicates significant difference between groups.

Abbreviations: $\mathrm{RT}$, reaction time; $\mathrm{Cl}$, confidence interval; $\mathrm{HC}$, healthy control; CFS, chronic fatigue syndrome; FM, fibromyalgia.

\section{Purdue pegboard test}

The FM group had the lowest mean score in this test, followed by CFS. The mean (SD) of inserted pins were 15.6 (1.1), 15.1 (1.7), and 14.7 (1.7) in the HC, CFS, and FM groups respectively. Moreover, HC, CFS, and FM groups had the range of 14-18, 12-18 and 11-18 scores respectively. There was no main effect of group, $\mathrm{F}(2,57)=1.71, p=0.191$, for the Purdue Pegboard test. The effect size was $\eta^{2}=0.06$, and observed power $=0.34$ for the group factor. Thus, no differences were found between the three groups. However, $20 \%$ of the FM patients and $15 \%$ of the CFS group performed below the threshold for poor performance for normal population $<60$ years of age. Additionally, Fisher's exact test showed no significant difference in the proportion ( $<14$ vs $\geq 14$ pins) between the groups $(p=0.12)$. A weak, negative correlation was found between the number of pins placed and the RT $(r=-0.22, p=0.09)$.

\section{Discussion}

Consistent with our hypothesis, the study revealed no significant difference between CFS and FM for motor proficiency in either gross or fine motor tasks. Both the FM and CFS groups showed significantly longer RT than the $\mathrm{HC}$ group in 
the gait initiation task, even after correcting for the potential effect of the participants' weight. In the Purdue Pegboard test, $20 \%$ in the FM group and $15 \%$ in the CFS group scored below the threshold for poor performance for their age group, whereas no one in the HC group scored below this threshold. However, statistical analyses did not show any significant differences between the groups in this task. The reduction in motor proficiency seems thus to be more noticeable in gross motor tasks than in fine motor tasks. Reduced movement velocity of upper extremity has been reported to be a strong determinant of reduced lower extremity velocity in the healthy elderly. ${ }^{23}$ Similarly, we found a weak, negative correlation between the number of inserted pins and RT. Thus, lower scores of inserted pins in some patients and longer RT in the gait initiation may possibly be interdependent (albeit weakly) in those individuals. Notably, the number of pins inserted in the Purdue Pegboard test did not differ statistically between three groups, and the sample size was small. Thus, a possible association has to be confirmed with a larger sample.

There are indications that high BMI has negative consequences on fine as well as gross motor skills. ${ }^{31}$ In the present study, the FM group had the highest BMI and weight, followed by the CFS and HC groups; the difference was not significant between the groups for BMI but it was for weight. The magnitude of BMI was moderated by the body height of the participants. High BMI is common both in FM and CFS populations, as well as low level of physical activity. ${ }^{32,33}$ The low level of physical activity is presumably explained by the habit of avoiding aggravation of pain and fatigue ${ }^{34}$ which consequently results in sedentariness and deconditioned muscles. ${ }^{35}$ Low level of physical activity may also be correlated with poor motor control and lower movement velocity. ${ }^{36}$ Low level of physical activity is furthermore shown to negatively influence manual discrete aiming. Even though age matters for performance, younger participants with a low level of physical activity displayed worse performance in manual dexterity with as well as without vision. ${ }^{37}$ Therefore, presumed low level of physical activity may have had an adverse effect on motor proficiency in the patients in the present study.

The FM group was significantly heavier than HC group, and weight was positively $(r=0.42)$ correlated with the RT during gait initiation. However, weight was not significantly correlated $(r=-0.17)$ with the raw scores of the Purdue Pegboard test. Thus, to compensate its potential effect, weight was adjusted for only in the analysis of RT between groups.

There are other important factors which may explain the poor motor function (especially gross motor function) in the patients. The interplay between sensory feedback and motor output is essential to produce smooth, coordinated movements and recognition of body position. ${ }^{38}$ Previous studies have shown deficits in the sensory-motor processing in FM and CFS at both peripheral and central levels of the central nervous system. ${ }^{39-42}$ This is hypothesized to be due to the longer time needed by the nervous system to acquire and process sufficient information related to sensory inputs as well as to produce appropriate movements. ${ }^{39,40}$ Moreover, there is evidence that experimental muscle pain can trigger inhibitory mechanisms and reduce the motor activity of the painful and other synergistic muscles. ${ }^{43}$ Since FM patients usually have higher pain levels relative to CFS patients, it is thus plausible to find worse motor performance in the FM than the CFS group. Although motor performance in general was worse in the FM group, we did not find any differences between patient groups, possibly due to small sample size. Another aspect of abnormalities in FM and CFS is the significant acceleration of age-related decrease in white and gray matter in the central nervous system. ${ }^{44,45}$ Accordingly, it is likely that the CFS and the FM patients show similar results as the elderly in motor tasks such as gait initiations and the Purdue Pegboard test. For instance, our finding of longer RT is in agreement with a previous study which showed that RT during gait initiation is longer in older compared to younger participants. $^{46}$

Accordingly, the observed impairments in the earlier studies may be responsible for a lower level of motor proficiency in the patients with CFS and FM. Therefore, patients may benefit from exercise therapy including sensory-motor challenges. In order to develop effective rehabilitation programs for this purpose, further research is needed to investigate the nature of motor control deficits as well as neural and muscular impairments in motor performance in these patients.

Some limitations of the present study should be considered for interpretation and implication of the findings. First, patients with FM in the present study were diagnosed according to the 1990 ACR criteria as these are used at the clinic. The revised 2010 criteria are expanded to include fatigue and cognition and no longer include digital palpation of tender points. Thus, patients diagnosed with FM according to the new criteria may be more similar to patients with CFS than those who were included in the present study. Second, the sample of twenty individuals in each group was relatively small. Third, the groups were not perfectly matched regarding weight and BMI. These discrepancies do however reflect the actual patient groups as mentioned in the discussion. Only women were included as most patients are female, ${ }^{24}$ and the 
results should not automatically be transferred to the whole population. Finally, medication usage was not specified and controlled for. As the patients usually take nonopioid analgesics when needed, this may potentially have affected their performance positively due to reducing pain or negatively due to a potential side effect of the medications.

\section{Conclusion}

The present study revealed no significant difference between CFS and FM for motor proficiency in either gross or fine motor tasks. Both CFS and FM groups showed longer RT in the gait initiation task compared to HC. The Purdue Pegboard test could not distinguish differences between the groups. Overall, it seems that gross motor function is more affected by FM and CFS than fine motor function.

\section{Acknowledgments}

The authors gratefully thank all the participants, the National Competence Centre for Complex Symptom Disorders, and the Norwegian Fibromyalgia Association for their cooperation. This study was funded by Sør-Trøndelag University College (HiST).

\section{Disclosure}

The authors report no conflicts of interest in this work.

\section{References}

1. White KP, Speechley M, Harth M. Co-existence of chronic fatigue syndrome with Fibromyalgia syndrome in the general population. Scand J Rheumatol. 2000;29:44-51.

2. Fukuda K, Straus SE, Hickie I, Sharpe MC, Dobbins JG, Komaroff A. The chronic fatigue syndrome: a comprehensive approach to its definition and study. International Chronic Fatigue Syndrome Study Group. Ann Intern Med. 1994;121(12):953-959.

3. Wolfe F, Clauw DJ, Fitzcharles MA, et al. The American College of Rheumatology preliminary diagnostic criteria for fibromyalgia and measurement of symptom severity. Arthritis Care Res (Hoboken). 2010;62(5):600-610.

4. Shafran SD. The chronic fatigue syndrome. Am JMed. 1991;90(6):730-739.

5. Wessely S, Nimnuan C, Sharpe M. Functional somatic syndromes: one or many? Lancet. 1999;354(9182):936-939.

6. Meeus M, Ickmans K, Struyf F, et al. What is in a name? Comparing diagnostic criteria for chronic fatigue syndrome with or without fibromyalgia. Clin Rheumatol. 2016;35(1):191-203.

7. Schmaling KB, Betterton KL. Neurocognitive complaints and functional status among patients with chronic fatigue syndrome and fibromyalgia. Qual Life Res. 2016;25(5):1257-1263.

8. Glass JM. Cognitive dysfunction in fibromyalgia and chronic fatigue syndrome: new trends and future directions. Curr Rheumatol Rep. 2006; $8(6): 425-429$.

9. Dick BD, Verrier MJ, Harker KT, Rashiq S. Disruption of cognitive function in fibromyalgia syndrome. Pain. 2008;139(3):610-616.

10. Majer M, Welberg LA, Capuron L, Miller AH, Pagnoni G, Reeves WC. Neuropsychological performance in persons with chronic fatigue syndrome: results from a population-based study. Psychosom Med. 2008;70(7):829-836.
11. Cockshell SJ, Mathias JL. Cognitive deficits in chronic fatigue syndrome and their relationship to psychological status, symptomatology, and everyday functioning. Neuropsychology. 2013;27(2):230-242.

12. Hou R, Moss-Morris R, Risdale A, et al. Attention processes in chronic fatigue syndrome: attentional bias for health-related threat and the role of attentional control. Behav Res Ther. 2014;52:9-16.

13. Leavitt F, Katz RS. Cognitive dysfunction in fibromyalgia: slow access to the mental lexicon. Psychol Rep. 2014;115(3):828-839.

14. Togo F, Lange G, Natelson BH, Quigley KS. Attention network test: assessment of cognitive function in chronic fatigue syndrome. J Neuropsychol. 2015;9(1):1-9.

15. Reyes Del Paso GA, Montoro CI, Duschek S. Reaction time, cerebral blood flow, and heart rate responses in fibromyalgia: evidence of alterations in attentional control. J Clin Exp Neuropsychol. 2015;37(4): 414-428.

16. Van Den Eede F, Moorkens G, Hulstijn W, et al. Psychomotor function and response inhibition in chronic fatigue syndrome. Psychiatry Res. 2011;186(2-3):367-372.

17. Veldhuijzen DS, Sondaal SF, Oosterman JM. Intact cognitive inhibition in patients with fibromyalgia but evidence of declined processing speed. J Pain. 2012;13(5):507-515.

18. Canny ML, Thompson JM, Wheeler MJ. Reliability of the box and block test of manual dexterity for use with patients with fibromyalgia. Am J Occup Ther. 2009;63(4):506-510.

19. Perez-de-Heredia-Torres M, Martinez-Piedrola RM, Cigaran-Mendez M, Ortega-Santiago R, Fernandez-de-Las-Penas C. Bilateral deficits in fine motor control ability and manual dexterity in women with fibromyalgia syndrome. Exp Brain Res. 2013;226(1):137-143.

20. Auvinet B, Chaleil D, Cabane J, et al. The interest of gait markers in the identification of subgroups among fibromyalgia patients. $B M C$ Musculoskelet Disord. 2011;12:258.

21. Pierrynowski MR, Tiidus PM, Galea V. Women with fibromyalgia walk with an altered muscle synergy. Gait Posture. 2005;22(3):210-218.

22. Paul L, Rafferty D, Wood L, Maclaren W. Gait characteristics of subjects with chronic fatigue syndrome and controls at self-selected and matched velocities. J Neuroeng. Rehabil. 2008;5:16.

23. Iwata A, Higuchi Y, Sano Y, et al. Maximum movement velocity of the upper limbs reflects maximum gait speed in community-dwelling adults aged older than 60 years. Geriatr Gerontol Int. 2014;14(4):886-891.

24. Wolfe F, Smythe HA, Yunus MB, et al. The American College of Rheumatology 1990 criteria for the classification of fibromyalgia. Report of the multicenter criteria committee. Arthritis Rheum. 1990;33(2): 160-172.

25. Chalder T, Berelowitz G, Pawlikowska T, et al. Development of a fatigue scale. J Psychosom Res. 1993;37(2):147-153.

26. Burckhardt CS, Clark SR, Bennett RM. The fibromyalgia impact questionnaire: development and validation. J Rheumatol. 1991;18(5): 728-733.

27. Martin K, Blizzard L, Garry M, Thomson R, McGinley J, Srikanth V. Gait initiation in older people-Time to first lateral movement may be the measure of choice. Gait Posture. 2011;34(3):374-378.

28. Tiffin J, Asher EJ. The purdue pegboard; norms and studies of reliability and validity. J Appl Psychol. 1948;32(3):234-247.

29. Causby R, Reed L, McDonnell M, Hillier S. Use of objective psychomotor tests in health professionals. Percept Mot Skills. 2014;118(3): $765-804$.

30. Stensdotter AK, Hodges P, Ohberg F, Hager-Ross C. Quadriceps EMG in open and closed kinetic chain tasks in women with patellofemoral pain. J Mot Behav. 2007;39(3):194-202.

31. Meng H, O'Connor DP, Lee BC, Layne CS, Gorniak SL. Effects of adiposity on postural control and cognition. Gait Posture. 2016;43:31-37.

32. Schmaling KB, Fiedelak JI, Bader J, Buchwald D. A longitudinal study of physical activity and body mass index among persons with unexplained chronic fatigue. J Psychosom Res. 2005;58(4):375-381.

33. McLoughlin MJ, Colbert LH, Stegner AJ, Cook DB. Are women with fibromyalgia less physically active than healthy women? Med Sci Sports Exerc. 2011;43(5):905-912. 
34. Nijs J, Roussel N, Van Oosterwijck J, et al. Fear of movement and avoidance behaviour toward physical activity in chronic-fatigue syndrome and fibromyalgia: state of the art and implications for clinical practice. Clin Rheumatol. 2013;32(8):1121-1129.

35. Lavergne MR, Cole DC, Kerr K, Marshall LM. Functional impairment in chronic fatigue syndrome, fibromyalgia, and multiple chemical sensitivity. Can Fam Physician. 2010;56(2):e57-e65.

36. Buchman AS, Boyle PA, Wilson RS, Bienias JL, Bennett DA. Physical activity and motor decline in older persons. Muscle Nerve. 2007;35(3): 354-362.

37. Van Halewyck F, Lavrysen A, Levin O, Boisgontier MP, Elliott D, Helsen WF. Both age and physical activity level impact on eye-hand coordination. Hum Mov Sci. 2014;36:80-96.

38. McCabe CS, Cohen H, Hall J, Lewis J, Rodham K, Harris N. Somatosensory conflicts in complex regional pain syndrome type 1 and fibromyalgia syndrome. Curr Rheumatol Rep. 2009;11(6):461-465.

39. McCabe CS, Cohen H, Blake DR. Somaesthetic disturbances in fibromyalgia are exaggerated by sensory motor conflict: implications for chronicity of the disease? Rheumatology (Oxford). 2007;46(10): 1587-1592.
40. Clauw DJ. Perspectives on fatigue from the study of chronic fatigue syndrome and related conditions. PMR. 2010;2(5):414-430.

41. Rasouli O, Stensdotter A, Van der Meer AL. TauG-guidance of dynamic balance control during gait initiation in patients with chronic fatigue syndrome and fibromyalgia. Clin Biomech. (Bristol, Avon). 2016;37:147-152.

42. Montoya P, Sitges C, Garcia-Herrera M, et al. Abnormal affective modulation of somatosensory brain processing among patients with fibromyalgia. Psychosom Med. 2005;67(6):957-963.

43. Madeleine P, Samani A, Binderup AT, Stensdotter AK. Changes in the spatio-temporal organization of the trapezius muscle activity in response to eccentric contractions. Scand J Med Sci Sports. 2011;21(2):277-286.

44. Kuchinad A, Schweinhardt P, Seminowicz DA, Wood PB, Chizh BA, Bushnell MC. Accelerated brain gray matter loss in fibromyalgia patients: premature aging of the brain? J Neurosci. 2007;27(15):4004-4007.

45. Puri BK, Jakeman PM, Agour M, et al. Regional grey and white matter volumetric changes in myalgic encephalomyelitis (chronic fatigue syndrome): a voxel-based morphometry 3 T MRI study. Br J Radiol. 2012;85(1015):e270-e273.

46. Henriksson M, Hirschfeld H. Physically active older adults display alterations in gait initiation. Gait Posture. 2005;21(3):289-296.

\section{Journal of Pain Research}

\section{Publish your work in this journal}

The Journal of Pain Research is an international, peer reviewed, open access, online journal that welcomes laboratory and clinical findings in the fields of pain research and the prevention and management of pain. Original research, reviews, symposium reports, hypothesis formation and commentaries are all considered for publication.

\section{Dovepress}

The manuscript management system is completely online and includes a very quick and fair peer-review system, which is all easy to use. Visit http://www.dovepress.com/testimonials.php to read real quotes from published authors. 